\title{
A fixed point theorem for smooth extension maps
}

\author{
Nirattaya Khamsemanan ${ }^{1 *}$, Robert F Brown ${ }^{2}$, Catherine Lee ${ }^{3}$ and Sompong Dhompongsa ${ }^{4}$
}

"Correspondence: nirattaya@siit.tu.ac.th

${ }^{1}$ Sirindhorn International Institute of Technology (SIIT), Thammasat University, Pathum Thani, Thailand Full list of author information is available at the end of the article

\begin{abstract}
Let $X$ be a compact smooth $n$-manifold, with or without boundary, and let $A$ be an $(n-1)$-dimensional smooth submanifold of the interior of $X$. Let $\phi: A \rightarrow A$ be a smooth map and $f:(X, A) \rightarrow(X, A)$ be a smooth map whose restriction to $A$ is $\phi$. If $p \in A$ is an isolated fixed point of $f$ that is a transversal fixed point of $\phi$, that is, the linear transformation $d \phi_{p}-I_{A}: T_{p} A \rightarrow T_{p} A$ is nonsingular, then the fixed point index of $f$ at $p$ satisfies the inequality $|i(X, f, p)| \leq 1$. It follows that if $\phi$ has $k$ fixed points, all transverse, and the Lefschetz number $L(f)>k$, then there is at least one fixed point of $f$ in $X \backslash A$. Examples demonstrate that these results do not hold if the maps are not smooth.

MSC: $55 \mathrm{M} 20 ; 54 \mathrm{C} 20$

Keywords: smooth manifold; smooth map; extension of a map; fixed point index; transversal fixed point; inverse function theorem; Lefschetz number; Lefschetz-Hopf theorem
\end{abstract}

\section{Introduction}

It has been known at least since the work of Shub and Sullivan in 1974 [1] that the values of the fixed point index of smooth maps are more restricted than they are for continuous functions in general. In [2] it is proved that, given integers $r$ and $s$, there is a map $f: X \rightarrow X$ of a manifold with boundary $\partial X$ that restricts to $\left.f\right|_{\partial X}=\phi: \partial X \rightarrow \partial X$ and an isolated fixed point $p$ of $f$ such that the fixed point indices are $i(\partial X, \phi, p)=r$ and $i(X, f, p)=s$. On the other hand, it is proved in that paper that if $f:(X, \partial X) \rightarrow(X, \partial X)$ is smooth and $p$ is a transverse fixed point of $\phi$, then either $i(X, f, p)=0$ or $i(X, f, p)=i(\partial X, \phi, p)$. A consequence of this result is that, under appropriate hypotheses on a smooth map $f$, it must have fixed points on $X \backslash \partial X$, the interior of the manifold $X$. Those same hypotheses are shown to be insufficient to imply the existence of such interior fixed points if the map $f$ is not smooth. (See also $[3,4]$.

We will consider a somewhat different setting, as follows. Let $X$ be a compact smooth $n$-manifold, with or without boundary, and let $A$ be a smooth (n-1)-dimensional submanifold of the interior of $X$. As in [2], we shall consider $f:(X, A) \rightarrow(X, A)$ to be an extension of its restriction $\left.f\right|_{A}=\phi: A \rightarrow A$. Suppose that $p$ is a transverse fixed point of $\phi$, then a simple example will show that the relationship between $i(X, f, p)$ and $i(A, \phi, p)$ cannot be as close as it is when $A=\partial X$. However, we will prove that there is still a very strong restriction on the value of $i(X, f, p)$, namely, that $|i(f, X, p)| \leq 1$. As a consequence, we obtain a condition on the Lefschetz number $L(f)$ of $f$ that implies the existence of fixed points of

\section{贷 Springer}

(02014 Khamsemanan et al.; licensee Springer. This is an Open Access article distributed under the terms of the Creative Commons Attribution License (http://creativecommons.org/licenses/by/2.0), which permits unrestricted use, distribution, and reproduction in any medium, provided the original work is properly cited. 
$f$ in $X \backslash A$. We demonstrate by an example that the same Lefschetz number condition is not sufficient to imply the existence of fixed points in $X \backslash A$ for maps $f:(X, A) \rightarrow(X, A)$ in general.

\section{The index of fixed points of smooth extension maps}

The index theorem of [2] is the following.

Theorem 1 Let $X$ be a compact smooth n-manifold with boundary $\partial X$. Given a smooth map $\phi: \partial X \rightarrow \partial X$ and a smooth map $f:(X, \partial X) \rightarrow(X, \partial X)$ extending $\phi$, suppose that $p \in$ $\partial X$ is an isolated fixed point of $f$ and that $d \phi_{p}-I_{\partial X}: T_{p}(\partial X) \rightarrow T_{p}(\partial X)$ is a nonsingular linear transformation. Then either $i(X, f, p)=0$ or $i(X, f, p)=i(\partial X, \phi, p)$.

We will modify the proof of Theorem 1 that was given in [2] in order to obtain a result of this type in the setting of smooth extension maps on a pair consisting of an $n$-dimensional compact smooth manifold $X$ and an $(n-1)$-dimensional smooth submanifold $A$ of the interior of $X$. Although our index result is similar to Theorem 1, the following example demonstrates that we cannot expect that there will be as close a relationship between the indices of $\phi$ and of $f$ as there is in Theorem 1. Let $f:\left(S^{2}, S^{1}\right) \rightarrow\left(S^{2}, S^{1}\right)$ where $S^{2}$ is viewed as the complex plane $\mathbb{C}$ compactified at infinity, $S^{1}$ is the unit circle, $f(z)=z^{2}$ for $z \in \mathbb{C}$ and $f(\infty)=\infty$. Let $\phi: S^{1} \rightarrow S^{1}$ be the restriction of $f$. Then $i\left(S^{1}, \phi, 1\right)=-1$, whereas $i\left(S^{2}, f, 1\right)=1$.

Theorem 2 Let $X$ be a smooth n-manifold, with or without boundary, and let $A$ be an $(n-1)$-dimensional smooth submanifold of the interior of $X$. Let $\phi: A \rightarrow A$ be a smooth map with smooth extension $f:(X, A) \rightarrow(X, A)$. Suppose that $p \in A$ is an isolated fixed point of $f$ and that $d \phi_{p}-I_{A}: T_{p} A \rightarrow T_{p} A$ is a nonsingular linear transformation. Then $|i(X, f, p)| \leq 1$.

Proof If the linear transformation $d f_{p}-I: T_{p} X \rightarrow T_{p} X$ is nonsingular, then $i(X, f, p)=$ \pm 1 , see [5]. Therefore, we assume that the linear transformation $d f_{p}-I: T_{p} X \rightarrow T_{p} X$ is singular. Note that determining the index of a $\operatorname{map} f$ at a fixed point $p$ is a local problem, so we may choose a local coordinate system about $p$ in which the smooth manifold $X$ is identified with $\mathbb{R}^{n}$ such that $p$ is the origin in $\mathbb{R}^{n}$ and the smooth submanifold $A$ is identified with the subspace $\mathbb{R}^{n-1}$.

Let us define $G: \mathbb{R}^{n} \rightarrow \mathbb{R}^{n}$ by $G(\mathbf{x})=f(\mathbf{x})-\mathbf{x}$. To calculate the index of $f$ at $p$, we need to determine the degree of $-G$ restricted to a sphere around $\mathbf{0}$. More specifically, for $\epsilon>0$ sufficiently small, we may consider the map $E_{\epsilon}: S^{n-1} \rightarrow \mathbb{R}^{n}-\{\mathbf{0}\}$ defined by

$$
E_{\epsilon}(\mathbf{x})=-G(\epsilon \mathbf{x}) .
$$

Since $G$ is a $C^{1}$ function with value $\mathbf{0}$ at the point $p$, we know from the definition of the derivative that

$$
\left|G(\epsilon \mathbf{x})-d G_{p}(\epsilon \mathbf{x})\right| \leq o(\epsilon)
$$

uniformly over $\{\mathbf{x} \in A|:| \mathbf{x} \mid=1\}$. Our goal is to define maps $P_{\epsilon}^{\sigma}$ related to $E_{\epsilon}$ such that $P_{\epsilon}^{\sigma}$ takes each of the upper and lower half-plane into either the upper or the lower half-plane 
and that will allow us to calculate the index $i(X, f, p)$. However, we need to make use of a change of coordinate. Since $d f_{p}-I=d G_{p}$ is singular but $\left.d G_{p}\right|_{A}$ is not, we know that $d G_{p}(0, \ldots, 0,1) \in \operatorname{span}\left\{x_{1}, \ldots, x_{n-1}\right\}$. Now rotate the $x_{1}, \ldots, x_{n-1}$ coordinate system so that $x_{n-1}$ points in the direction of $d G_{p}(0, \ldots, 0,1)$. Choose new coordinates $y_{1}, \ldots, y_{n}$ so that the hyperplane $y_{n}=0$ is the same as $x_{n}=0$ and, for each $j=1, \ldots, n-1$, the transformation $d G_{p}$ takes the $x_{j}$ unit vector to the $y_{i}$ unit vector. Now we have

$$
\begin{aligned}
& \left\{\left(x_{1}, \ldots, x_{n-1}, 0\right) \in \mathbb{R}^{n}\right\}=\left\{\left(y_{1}, \ldots, y_{n-1}, 0\right) \in \mathbb{R}^{n}\right\}, \\
& \left\{\left(x_{1}, \ldots, x_{n-1}, x_{n}\right) \in \mathbb{R}^{n}\right\}=\left\{\left(y_{1}, \ldots, y_{n-1}, y_{n}\right) \in \mathbb{R}^{n}\right\}
\end{aligned}
$$

and the positive $y_{n}$ axis is in the same half-space as the positive $x_{n}$ axis. With this change of coordinates, $d G_{p}$, as a map from $x$ coordinates into $y$ coordinates, has the following form:

$$
d G_{p}\left(\alpha_{1}, \ldots, \alpha_{n-1}, \alpha_{n}\right)=\left(\alpha_{1}, \ldots, \alpha_{n-1}, 0\right)+\alpha_{n}(0, \ldots, 0, B, 0)
$$

for some constant $B$. Define

$$
S_{+}=\left\{\left(x_{1}, \ldots, x_{n}\right) \in \mathbb{R}^{n}: x_{n} \geq 0\right\} \cap S^{n-1}, \quad S_{-}=\left\{\left(x_{1}, \ldots, x_{n}\right) \in \mathbb{R}^{n}: x_{n} \leq 0\right\} \cap S^{n-1}
$$

It is easy to check the following:

(i) The point

$$
\mathbf{x}^{+}=\left(0, \ldots, 0,-\frac{B}{\left(1+B^{2}\right)^{\frac{1}{2}}}, \frac{1}{\left(1+B^{2}\right)^{\frac{1}{2}}}\right)
$$

is the unique point of $S_{+}$with the property that $d G_{p}\left(\mathbf{x}^{+}\right)=\mathbf{0}$. From the inverse function theorem, there is a small neighborhood $U^{+}$of the point $\mathbf{x}^{+}$such that $\left.d G_{p}\right|_{S_{+}}$is a nonsingular diffeomorphism of $U^{+}$onto a neighborhood of $\mathbf{0}$ in the hyperplane $y_{n}=0$ and $\left(\left.d G_{p}\right|_{S_{+}}\right)^{-1}\left(d G_{p}\left(U^{+}\right)\right)=U^{+}$.

(ii) The point

$$
\mathbf{x}^{-}=\left(0, \ldots, 0,-\frac{B}{\left(1+B^{2}\right)^{\frac{1}{2}}},-\frac{1}{\left(1+B^{2}\right)^{\frac{1}{2}}}\right)
$$

is the unique point of $S_{-}$with the property that $d G_{p}\left(\mathbf{x}^{-}\right)=\mathbf{0}$. Similarly, from the inverse function theorem, there is a small neighborhood $U^{-}$of the point $\mathbf{x}^{-}$such that $\left.d G_{p}\right|_{S_{-}}$is a nonsingular diffeomorphism of $U^{-}$onto a neighborhood of $\mathbf{0}$ in the hyperplane $y_{n}=0$ and $\left(\left.d G_{p}\right|_{S_{-}}\right)^{-1}\left(d G_{p}\left(U^{-}\right)\right)=U^{-}$.

Now consider the maps $P_{\epsilon}$ defined by

$$
P_{\epsilon}(\mathbf{x})=\epsilon^{-1} E_{\epsilon}(\mathbf{x}) .
$$

The map $\left.P_{\epsilon}\right|_{S^{n-1}}$ converges in the $C^{1}$ topology to $-\left.d G_{p}\right|_{S^{n-1}}$ because

$$
\begin{aligned}
d\left(P_{\epsilon}(\mathbf{x})\right) & =d\left(\epsilon^{-1} E_{\epsilon}(\mathbf{x})\right) \\
& =d\left(\epsilon^{-1}(-G(\epsilon \mathbf{x}))\right)
\end{aligned}
$$




$$
\begin{aligned}
& =-\epsilon^{-1} d(G(\epsilon \mathbf{X})) \\
& =-\epsilon^{-1} d G_{p}(\epsilon \mathbf{x}) \epsilon \\
& =-d G_{p}(\epsilon \mathbf{x}) .
\end{aligned}
$$

Consequently, we have the following analogues of (i)-(ii) above.

(1) There is a unique point $\mathbf{x}_{\epsilon}^{+} \in S_{+}$such that the $y_{1}, \ldots, y_{n-1}$ coordinates of $P_{\epsilon}\left(\mathbf{x}_{\epsilon}^{+}\right)$are all 0 . Also, since $p$ is an isolated fixed point of $f, P_{\epsilon}\left(\mathbf{x}_{\epsilon}^{+}\right) \neq \mathbf{0}$. In particular, the $y_{n}$ coordinate of $P_{\epsilon}\left(\mathbf{x}_{\epsilon}^{+}\right)$is nonzero. Hence we have

$$
P_{\epsilon}\left(\mathbf{x}_{\epsilon}^{+}\right)=\left(0, \ldots, 0, y_{n}^{\epsilon^{+}}\right)
$$

where $y_{n}^{\epsilon^{+}} \neq 0$.

(2) There is a unique point $\mathbf{x}_{\epsilon}^{-} \in S_{-}$such that the $y_{1}, \ldots, y_{n-1}$ coordinates of $P_{\epsilon}\left(\mathbf{x}_{\epsilon}^{-}\right)$are all 0 . Also, since $p$ is an isolated fixed point of $f, P_{\epsilon}\left(\mathbf{x}_{\epsilon}^{-}\right) \neq \mathbf{0}$. In particular, the $y_{n}$ coordinate of $P_{\epsilon}\left(\mathbf{x}_{\epsilon}^{-}\right)$is nonzero. Hence we have

$$
P_{\epsilon}\left(\mathbf{x}_{\epsilon}^{-}\right)=\left(0, \ldots, 0, y_{n}^{\epsilon^{-}}\right)
$$

where $y_{n}^{\epsilon^{-}} \neq 0$.

If $P_{\epsilon}(\mathbf{x})=\left(y_{1}, y_{2}, \ldots, y_{n}\right)$, define a map $P_{\epsilon}^{\sigma}: S^{n-1} \rightarrow \mathbb{R}^{n}-\{\mathbf{0}\}$ by

$$
P_{\epsilon}^{\sigma}= \begin{cases}\left(y_{1}, \ldots, y_{n-1}, \sigma^{+}\left|y_{n}\right|\right) & \text { if } \mathbf{x} \in S_{+}, \\ \left(y_{1}, \ldots, y_{n-1}, \sigma^{-}\left|y_{n}\right|\right) & \text { if } \mathbf{x} \in S_{-} \backslash \mathbb{R}^{n-1}\end{cases}
$$

where $\sigma^{+}=y_{n}^{\epsilon^{+}} /\left|y_{n}^{\epsilon^{+}}\right|= \pm 1$ and $\sigma^{-}=y_{n}^{\epsilon^{-}} /\left|y_{n}^{\epsilon^{-}}\right|= \pm 1$.

Notice that the values of $\left.P_{\epsilon}^{\sigma}\right|_{S_{+}}$lie entirely in the half-space where $P_{\epsilon}\left(\mathbf{x}_{\epsilon}^{+}\right)$is located with respect to the $y_{1}, \ldots, y_{n}$ coordinates and the values of $\left.P_{\epsilon}^{\sigma}\right|_{S_{-}}$lie entirely in the half-space where $P_{\epsilon}\left(\mathbf{x}_{\epsilon}^{-}\right)$is located with respect to the $y_{1}, \ldots, y_{n}$ coordinates. Furthermore, $P_{\epsilon}$ and $P_{\epsilon}^{\sigma}$ are homotopic as maps into $\mathbb{R}^{n}-\{\mathbf{0}\}$ by the following homotopy:

$$
H(\mathbf{x}, t)=t P_{\epsilon}(\mathbf{x})+(1-t) P_{\epsilon}^{\sigma}(\mathbf{x})
$$

For $\mathbf{x} \in S_{+}$, either $\left(y_{1}, \ldots, y_{n-1}\right) \neq \mathbf{0}$ or $\mathbf{x}=\mathbf{x}_{\epsilon}^{+}$. If $\left(y_{1}, \ldots, y_{n-1}\right) \neq \mathbf{0}$, then $H(\mathbf{x}, t) \neq \mathbf{0}$ by definition. If $\mathbf{x}=\mathbf{x}_{\epsilon}^{+}$, then $H(\mathbf{x}, t)=P_{\epsilon}(\mathbf{x})$ which we know is never $\mathbf{0}$. A similar argument shows that $H(\mathbf{x}, t) \neq \mathbf{0}$ for $\mathbf{x} \in S_{-} \backslash \mathbb{R}^{n-1}$.

Since the map $P_{\epsilon}^{\sigma}$ takes $S_{+}$into either the upper or lower half-planes with respect to the $y$ coordinates and takes $S_{-}$into either the upper or lower half-planes with respect to the $y_{1}, \ldots, y_{n}$ coordinates, the map $P_{\epsilon}^{\sigma}$ is homotopic either to a constant map, the suspension of $\left.P_{\epsilon}^{\sigma}\right|_{S^{n-2}}=\left.P_{\epsilon}\right|_{S^{n-2}}$ or the suspension of $\left.P_{\epsilon}^{\sigma}\right|_{S^{n-2}}=\left.P_{\epsilon}\right|_{S^{n-2}}$ followed by a reflection about the hyperplane $y_{n}=0$. This homotopy tells us that either $P_{\epsilon}$ has degree 0 or $\operatorname{deg}\left(\left.P_{\epsilon}\right|_{S^{n-2}}\right)$ or $-\operatorname{deg}\left(\left.P_{\epsilon}\right|_{S^{n-2}}\right)$.

Although $P_{\epsilon}$ is a map from $x$ to $y$ coordinates, we know that the $x$ and $y$ coordinates are related by a linear map, call it $L$, satisfying $L\left(x_{i}\right)=y_{i}$, for $i=1, \ldots, n$. Thus, the map $L^{-1} \circ P_{\epsilon}$ 
takes $x_{1}, \ldots, x_{n}$ coordinates to $x_{1}, \ldots, x_{n}$ coordinates and

$$
\operatorname{deg}\left(L^{-1} \circ P_{\epsilon}\right)=\operatorname{deg}\left(L^{-1}\right) \cdot \operatorname{deg}\left(P_{\epsilon}\right)=\operatorname{deg}\left(P_{\epsilon}\right)
$$

Thus the index $i(X, f, p)=\operatorname{deg}\left(P_{\epsilon}\right)$ which is either 0 or \pm 1 .

\section{Fixed point theorem for smooth extension maps}

We can now use Theorem 2 to establish the existence of fixed points in $X \backslash A$.

Theorem 3 Suppose that $A$ is an $(n-1)$-dimensional smooth submanifold of the interior of a compact smooth n-manifold $X$. Given a smooth map $\phi: A \rightarrow A$ and a smooth map $f:(X, A) \rightarrow(X, A)$ extending $\phi$, suppose that the fixed points of $\phi$ are $\left\{x_{1}, x_{2}, \ldots, x_{k}\right\}$, all of which are transversal, that is, the linear map $d \phi_{x_{j}}-I_{A}$ is nonsingular for each $x_{j}$. If the Lefschetz number $L(f)>k$, then there must be at least one fixed point in $X \backslash A$.

Proof Suppose that $f$ only has fixed points in $A$. This means the fixed point set of $f$ is $\left\{x_{1}, \ldots, x_{k}\right\}$, the set of fixed points of $\phi$. Then, by the Lefschetz-Hopf theorem [6], the Lefschetz number of $f$ is

$$
L(f)=\sum_{j=0}^{k} i\left(X, f, x_{j}\right) \leq k
$$

because $i\left(X, f, x_{j}\right) \leq 1$ by Theorem 2 . This is contrary to the assumption that $L(f)>k$, so $f$ has fixed points in $X \backslash A$.

A consequence of this theorem is the following.

Corollary 4 Let $S^{2}$ be the complex plane $\mathbb{C}$ compactified at infinity and $S^{1}$ be the unit circle. Suppose that $\phi: S^{1} \rightarrow S^{1}$ is a smooth map defined by $\phi(\zeta)=\zeta^{k}$ for some $k \geq 2$ and that $f:\left(S^{2}, S^{1}\right) \rightarrow\left(S^{2}, S^{1}\right)$ is a smooth extension of $\phi$. Iff is homotopic to the suspension of $\phi$, then there is at least one fixed point in $S^{2} \backslash S^{1}$.

Proof Since $f$ is homotopic to the suspension of $\phi$, then $f$ is of degree $k$ and thus $L(f)=$ $1+k$. However, $\phi$ has only $k-1$ fixed points. Note here that the $k-1$ fixed points of $\phi$ on $S^{1}$ are all transversal so that the hypotheses of Theorem 3 hold.

The following example illustrates the fact that the corollary, and therefore Theorem 3, require the hypothesis that the map $f$ is smooth, by exhibiting a non-smooth map $f:\left(S^{2}, S^{1}\right) \rightarrow\left(S^{2}, S^{1}\right)$ homotopic to the suspension of $\phi$ that has no fixed points on $S^{2} \backslash S^{1}$.

Example 1 Let $S^{2}=\mathbb{C} \cup\{\infty\}$ be the complex plane $\mathbb{C}$ compactified at infinity and $S^{1}$ be the unit circle. Let $\phi: S^{1} \rightarrow S^{1}$ be defined by $\phi(\zeta)=\zeta^{2}$. Let $B_{+}^{2}=\{z \in \mathbb{C}:|z| \leq 1\}$ and $B_{-}^{2}=$ $\{z \in \mathbb{C}:|z| \geq 1\} \cup\{\infty\}$, so $S^{2}=B_{+}^{2} \cup B_{-}^{2}$ and $B_{+}^{2} \cap B_{-}^{2}=S^{1}$. For $z \in B_{-}^{2} \backslash\{1\}$, there exist $\zeta \in S^{1}$ and $t \in[0,1)$ such that $z=t 1+(1-t) \zeta$. As in Lemma 6.1 of [7], define $f: B_{+}^{2} \rightarrow B_{+}^{2}$ by setting $f(z)=t 1+(1-t) \phi(\zeta)$ for $z \neq 1$ and $f(1)=1$, then $f$ has no fixed points in $B_{+}^{2} \backslash S^{1}$. In order to extend $f$ to a selfmap of $B_{-}^{2}$ so that there are no fixed points on $B_{-}^{2} \backslash S^{1}$, we define $\rho: S^{2} \rightarrow S^{2}$ by $\rho(z)=\rho\left(r e^{i \theta}\right)=\frac{1}{r} e^{i \theta}, \rho(0)=\infty$ and $\rho(\infty)=0$, so $\rho\left(B_{-}^{2}\right)=B_{+}^{2}$ and $\rho\left(B_{+}^{2}\right)=B_{-}^{2}$. 
Then, for $z \in B_{-}^{2}$, let $f(z)=\rho f \rho(z)$. The only fixed point of $f$ is $z=1$ and thus there are no fixed points in $S^{2} \backslash S^{1}$.

\section{Competing interests}

The authors declare that they have no competing interests.

\section{Authors' contributions}

All authors contributed equally to the writing of this paper. All authors read and approved the final manuscript.

\section{Author details}

'Sirindhorn International Institute of Technology (SIIT), Thammasat University, Pathum Thani, Thailand. ${ }^{2}$ Department of Mathematics, University of California, Los Angeles, USA. ${ }^{3}$ School of Public Health, Harvard University, Cambridge, USA.

${ }^{4}$ Department of Mathematics, Chiang Mai University, Chiang Mai, Thailand.

\section{Acknowledgements}

This work has been supported by the Thailand Research Fund MRG5580232 under the mentorship of Professor Robert F. Brown of the Department of Mathematics, UCLA and Professor Sompong Dhompongsa of the Department of Mathematics, Chiang Mai University. The author would also like to thank the referee for all the useful suggestions.

\section{Received: 26 December 2013 Accepted: 31 March 2014 Published: 16 Apr 2014}

\section{References}

1. Shub, M, Sullivan, D: A remark on the Lefschetz fixed point formula for differentiable maps. Topology 13, 189-191 (1974)

2. Brown, RF, Greene, RE, Schirmer, H: Fixed points of map extensions. In: Topological Fixed Point Theory and Applications (Tianjin, 1988). Lecture Notes in Math., vol. 1411, pp. 24-45. Springer, Berlin (1989)

3. Brown, RF, Greene, RE: An interior fixed point property of the disc. Am. Math. Mon. 101(1), 39-47 (1994)

4. Schirmer, H: Nielsen theory of transversal fixed point sets. Fundam. Math. 141(1), 31-59 (1992). With an appendix by Robert E Greene

5. Guillemin, V, Pollack, A: Differential Topology. Am. Math. Soc., Providence (1974)

6. Arkowitz, M, Brown, RF: The Lefschetz-Hopf theorem and axioms for the Lefschetz number. Fixed Point Theory Appl. 2004, Article ID 465090 (2004)

7. Schirmer, H: A relative Nielsen number. Pac. J. Math. 122, 459-473 (1986)

10.1186/1687-1812-2014-97

Cite this article as: Khamsemanan et al.: A fixed point theorem for smooth extension maps. Fixed Point Theory and Applications 2014, 2014:97

\section{Submit your manuscript to a SpringerOpen ${ }^{\circ}$ journal and benefit from:}

- Convenient online submission

- Rigorous peer review

- Immediate publication on acceptance

Open access: articles freely available online

- High visibility within the field

- Retaining the copyright to your article 